Arq. Bras. Med. Vet. Zootec., v.70, n.1, p.45-52, 2018

\title{
Metaloproteinases no tecido laminar do casco de equinos submetidos à obstrução intraluminal do cólon menor
}

\author{
[Metalloproteinases in hoof laminar tissue of equine submitted to \\ intraluminal small colon obstruction]
}

\author{
V.A. Canello ${ }^{1}$, K. Gravena ${ }^{1}$, M.C.H. Tovar ${ }^{1}$, D.P.M. Dias ${ }^{1}$, L.M.W. Gomide ${ }^{1}$, N.S. Bernardi ${ }^{1}$, \\ A.F. Sabes ${ }^{1}$, D.J. Queiroz ${ }^{1}$, G.D. Melo ${ }^{2}$, G.F. Machado ${ }^{2}$, J.C. Lacerda Neto ${ }^{1}$ \\ ${ }^{1}$ Faculdade de Ciências Agrárias e Veterinárias - FCAV-Unesp - Jaboticabal, SP \\ ${ }^{2}$ Faculdade de Medicina Veterinária - FMVA-Unesp - Araçatuba, SP
}

\begin{abstract}
RESUMO
O objetivo deste estudo foi avaliar a expressão das MMP-2 e MMP-9 no tecido laminar do casco e o perfil leucocitário de equinos submetidos à obstrução intraluminal do cólon menor. Realizaram-se laparotomia e obstrução do cólon menor de oito equinos hígidos, utilizando-se uma bola inserida no lúmem intestinal. A bola foi inflada à pressão de $80 \mathrm{mmHg}$ e a obstrução foi mantida por quatro horas. Foram realizadas coletas sanguíneas antes da obstrução (M0), imediatamente após a desobstrução (M4) e a cada 12 horas após M4, até completar 72 horas (M12, M24, M36, M48, M60 e M72). As biópsias de casco foram realizadas em M0, M4 e M72, e as amostras foram submetidas à análise zimográfica. Foi observado aumento nos leucócitos em M12 e M24, decorrente do aumento de neutrófilos segmentados e bastonetes, os quais diminuíram a partir de M36. Segundo a técnica zimográfica, não se observaram alterações nos valores de MMP-2 e -9, possivelmente devido à baixa intensidade das lesões ocasionadas no cólon menor. Com isso, conclui-se que as alterações inflamatórias decorrentes da obstrução do cólon menor não foram suficientes para ocasionar alterações na expressão das MMP-2 e -9 no tecido laminar podal.
\end{abstract}

Palavras-chave: biópsia podal, cólica, laminite, matriz metaloproteinase

\begin{abstract}
The aim of this study was to evaluate the blood leukocytes and the MMP-2 and -9 expression in the hoof laminar tissue of horses undergoing intraluminal small colon obstruction. Laparotomy and the small colon obstruction was performed in eight healthy horses, inserting a ball in the intestinal lumen. The ball was inflated to $80 \mathrm{mmHg}$ pressure and the occlusion was maintained for 4 hours. The blood was collectedBlood samples were taken before the obstruction (M0), immediately after intestinal clearance (M4), and every 12 hours until completeuntil 72 hours (M12, M24, M36, M48, M60 and M72). The hoof biopsies were performed at M0, M4, and M72 and the samples were subjected to zymography analysis. There was an increase in leukocytes in M12 and M24, due to the increase in segmented neutrophils and band neutrophils, which decreased as of M36. According to zymography technique not observed changes were not not observed in MMP-2 and -9, possibly due to the low intensity of the small colon lesions. Wherefore, it is concludedIn conclusion, that the inflammatory changes resulting from small colon obstruction were not enough to cause changes in the expression of MMP-2 and -9 in the hoof laminar tissue.
\end{abstract}

Keywords: hoof biopsy, colic, laminitis, matrix metalloproteinases

Recebido em 11 de agosto de 2016

Aceito em 3 de fevereiro de 2017

E-mail: vinaocanello@hotmail.com 


\section{INTRODUÇÃO}

Em milhões de anos de evolução, o dígito dos equinos passou por diversas transformações, tornando-se uma estrutura complexa, resistente e adaptada às mais variadas condições ambientais e topográficas, o que teve grande importância para a sobrevivência da espécie (Pollitt, 2004). Entretanto, desde o início da domesticação, diversas alterações impostas pelo homem ao modo de vida dos equinos acabaram por tornar o casco susceptível a várias afecções, entre elas a laminite (Pollitt, 1990). Diversos estudos buscam elucidar as causas da laminite, entre os quais alguns sugerem estreita relação entre a ocorrência de cólica e o desenvolvimento de laminite (Mungall e Pollitt, 1999; Visser e Pollitt, 2011; Belknap e Black, 2012).

A cólica equina se caracteriza pela dor abdominal, que pode se manifestar de forma aguda ou crônica, e tem como causas comuns distensão intestinal, vólvulos, além de úlceras no estômago ou no intestino (Magdesian e Smith, 2006). Nos casos com acometimento do intestino grosso $(19 \%), 51,35 \%$ são ocasionadas por obstrução do cólon menor (Corrêa et al., 2006). Essa distensão intraluminal promove isquemia mediante a redução da perfusão microvascular na parede intestinal (Faleiros et al., 2002), que pode ser suficiente para causar lesões na camada seromuscular e lesões remotas nos pulmões, ambas características do processo de isquemia e reperfusão (Faleiros et al., 2008), processo esse relacionado a complicações pós-cirúrgicas, entre as quais também se podem destacar a endotoxemia e a laminite (Moore et al., 1995).

A laminite equina é caracterizada pela degeneração das lâminas dérmicas e epidérmicas, o que faz com que elas se separem, levando à perda da coesão entre a falange distal e a muralha do casco. Essa coesão se deve principalmente à existência de placas de adesão denominadas hemidesmossomos, constituídas por agregados proteicos que formam ligações entre o interior celular e o exterior, composto de tecido conjuntivo (Pollitt, 2004). Em condições fisiológicas, essa coesão se remodela de forma constante, permitindo, assim, o crescimento e a regeneração do casco. Isso ocorre devido à ativação controlada de matriz de metaloproteinases (MMP) (Woessner, 1991). A ação proteolítica das MMP no processo de remodelação é controlada pelos inibidores teciduais das MMP (TIMP), que evitam a degradação excessiva da matriz extracelular, de modo que o desequilíbrio entre as MMP e seus respectivos TIMP pode levar ao surgimento de lesões na estrutura celular (Vu e Werb, 2000; Raffetto e Khalil, 2008). Desse modo, a ação exacerbada e descontrolada das MMP está diretamente relacionada ao desenvolvimento de inúmeras enfermidades, como, por exemplo, a laminite (Clutterbuck et al., 2010).

As MMP são secretadas como pró-enzimas inativas (pró-MMP) por neutrófilos, macrófagos, fibroblastos e células tumorais, em resposta a diferentes estímulos de natureza variada (Woessner, 1991). A ativação se dá por meio de processo de clivagem proteolítica (Visse e Nagase, 2003), e a expressão das MMP pode ser regulada por citocinas pró-inflamatórias em diversos tipos de tecido, o que sugere relação entre o processo inflamatório e as MMP (Clutterbuck et al., 2010).

Nos casos de laminite, a degradação laminar podal causada pelas MMP está associada à liberação de fatores de ativação da corrente sanguínea para os dígitos (Clutterbuck et al., 2010). Alguns autores, como Pollitt et al. (1998) e Medina-Torres et al. (2011), evidenciaram aumento na expressão e ativação das MMP-2 e 9 em estudos com laminite.

Diante disso, levando em consideração o processo isquêmico e a resposta inflamatória induzida pelos distúrbios gastrointestinais, bem como sua repercussão sistêmica, este trabalho busca relacioná-los ao desenvolvimento da laminite, a fim de avaliar a possível elevação nos valores da expressão e ativação das MMP-2 e 9 nos tecidos dérmicos e epidérmicos lamelares em equinos submetidos à obstrução intraluminal do cólon menor.

\section{MATERIAL E MÉTODOS}

Este trabalho de pesquisa foi aprovado pelo Comitê de Ética no Uso de Animais (Ceua) da Faculdade de Ciências Agrárias e VeterináriasFCAV-Unesp, campus de Jaboticabal, sob o número de protocolo $007568-09$, em atendimento à lei $\mathrm{n}^{\mathrm{o}} 11.794$, de 8 de outubro de 2008 . 
Foram utilizados oito equinos, quatro machos e quatro fêmeas, sem raça definida, com idade de $11 \pm 2$ anos e pesando $370,5 \pm 17,4 \mathrm{~kg}$, em bom estado nutricional e considerados hígidos após avaliação clínica e radiológica.

Após jejum alimentar e hídrico de 24 e 12 horas, respectivamente, esses animais foram anestesiados e submetidos à celiotomia e à obstrução intraluminal do cólon menor, de acordo com técnica descrita por Gravena et al. (2016). Como medicação pré-anestésica, utilizou-se cloridrato de detomidina $10 \%$; após 10 minutos, realizou-se indução anestésica com cetamina $10 \%$, seguida da administração de maleato de midazolam. A anestesia inalatória foi mantida com isoflurano vaporizado em oxigênio, em circuito semifechado, sob ventilação controlada. A celiotomia foi realizada por meio de incisão mediana pré-umbilical. Após esse procedimento, identificou-se o segmento do cólon a ser obstruído, e a borda antimesentérica foi incisada para a introdução da bola de $13 \mathrm{~cm}$ de diâmetro (Water Polo Nabaiji, Decathlon, Villeneuve-d'Ascq, France), a qual foi inflada até atingir a pressão de $80 \mathrm{mmHg}$. Os animais foram mantidos sob anestesia inalatória e obstruídos durante quatro horas, momento esse em que a bola foi desinflada e removida do lúmen intestinal.

As amostras de sangue venoso foram obtidas por meio de cateter (BD Insyte ${ }^{\circledR}$ Autoguard ${ }^{\circledR}$ 20G. BD-Becton Dickinson Indústrias Cirúrgicas S.A., Brasil), implantado na veia jugular externa esquerda, imediatamente antes da indução anestésica, para a realização da obstrução intraluminal do cólon menor (M0), logo após a desobstrução do cólon menor (M4) e, posteriormente, a cada 12 horas, durante o póscirúrgico, até que se completassem 72 horas após a desobstrução do cólon menor (M12, M24, M36, M48, M60 e M72). As amostras obtidas foram armazenadas em tubos de coleta a vácuo (ácido etilenodiaminotetracético) para a realização imediata das análises leucométricas.

As análises leucométricas foram realizadas em contador de células automático (Pock-100 iV Diff $^{\circledR}$, Sysmex do Brasil Indústria e Comércio Ltda. - Brasil), e as contagens diferenciais dos leucócitos sanguíneos foram realizadas em esfregaços corados com May-Grunwald-Giemsa. Após a determinação da contagem diferencial, os valores percentuais foram multiplicados pela contagem de leucócitos, o que definiu as concentrações absolutas dos diversos leucócitos.

Os animais foram submetidos a exame clínico em M0 e, posteriormente, a cada 12 horas, até que se completassem 72 horas (M12, M24, M36, M48, M60 e M72), avaliando-se frequências cardíaca (FC) e respiratória (FR); perfusão das mucosas, classificadas de acordo com a coloração e tempo de preenchimento capilar (TPC); apreciação da atividade intestinal mediante auscultação abdominal; avaliação do turgor cutâneo; determinação da temperatura retal (TR); qualidade do pulso das artérias digitais palmares, além da observação de sinais clínicos característicos referentes a desconforto abdominal.

As amostras de tecido laminar do casco foram coletadas em M0, M4 e M72, por meio da técnica descrita por Gravena et al. (2012). Os locais foram padronizados a $2 \mathrm{~cm}$ da borda coronária, e as amostras foram coletadas mediante aberturas de aproximadamente $20 \mathrm{~mm}$ de diâmetro, previamente realizadas na parede do casco. As amostras obtidas passaram por processo de congelamento rápido em nitrogênio líquido e posteriormente foram armazenadas a $70^{\circ} \mathrm{C}$, até a realização das análises zimográficas.

Para as análises das MMP-2 e -9 , realizou-se a avaliação zimográfica de acordo com a técnica descrita por Machado et al. (2010), com modificações. Inicialmente as amostras de tecido laminar foram maceradas juntamente com tampão de lise (Tris 50mM - 6,057g, NaCl $150 \mathrm{mM}-8,775 \mathrm{~g}, \mathrm{CaCl}_{2} 5 \mathrm{mM}-0,555 \mathrm{~g}$, éter dodecil-polioxietilenoglicol $0,05 \%$ (Brij-35) $0,5 \mathrm{~g}$, água destilada e tablete inibidor de protease), para a extração das proteínas. Após a realização da eletroforese, os géis corados foram fotografados em fotodocumentador de alta resolução e posteriormente analisados por meio do software ImageJ (Image processing and analysis in Java. National Institutes of Health, EUA), que analisou a densidade integrada das bandas digeridas no gel e expressou os valores em unidades arbitrárias.

A identificação das MMP nas suas formas ativas e inativas foi efetuada com base nos pesos moleculares de amostras controle (adenocarcinoma mamário canino), sendo, pró- 
MMP-9 (92kDa), MMP-9-ativa (86kDa), próMMP-2 (72kDa) e MMP-2-ativa (66kDa).

Os dados obtidos foram analisados segundo delineamento em blocos ao acaso, utilizando-se o programa computacional AgroEstat (AgroStatSistema para Análises Estatísticas de Ensaios Agronômicos, versão 1.1.0.626, 2011). As variáveis passaram inicialmente por teste de normalidade Kolmorogov-Smirnov e, posteriormente, por análise de variância (ANOVA). Os valores médios foram comparados pelo teste de Tukey, estabelecendose o nível de significância igual a $\mathrm{P}<0,05$.

\section{RESULTADOS}

À análise das variáveis clínicas, é possível observar que a FC, a FR e a TR apresentaram diferença significativa no decorrer do período experimental. A FC aumentou 12 horas após M4 $(\mathrm{P}<0,05)$, porém retornou aos valores basais após 24 horas, mantendo-se ligeiramente aumentada $(\mathrm{P}>0,05)$ até $\mathrm{M} 72$. A FR se elevou após 24 horas $(\mathrm{P}<0,05)$, retornando aos valores basais após 36 horas. A TR após 12 horas apresentou-se aumentada $(\mathrm{P}<0,05)$, voltando aos valores basais após 24 horas, e manteve-se constante até M72.

O TPC, a motilidade intestinal, o turgor cutâneo, a coloração de mucosa e o pulso das artérias digitais palmares não apresentaram diferenças significativas. Porém, observou-se que o TPC diminuiu inicialmente em M12, com posterior aumento em M60. A motilidade intestinal também apresentou alterações no decorrer do período experimental, apresentando-se diminuída em M12 e retornando aos valores basais em M60.

Os valores do leucograma no período póscirúrgico indicam aumento de leucócitos em M12 e M24 $(\mathrm{P}<0,05)$. Essa resposta leucocitária diminuiu a partir de M36 e manteve-se acima dos valores basais, porém o aumento não foi significativo. Já os eosinófilos apresentaram diminuição em seus valores em M12 e M36, com os demais momentos não diferindo do basal. Quanto aos bastonetes, esses aumentaram significativamente em M12 e M24, enquanto nos demais momentos se mantiveram inalterados em relação ao basal. Os neutrófilos segmentados aumentaram $(\mathrm{P}<0,05)$ em M12 e M36 e retornaram aos valores basais em M48. Houve também aumento discreto $(\mathrm{P}<0,05)$ de neutrófilos segmentados em M60, porém em M72 os valores retornaram àqueles registrados no basal (M0). Quanto aos linfócitos, registrouse diminuição $(\mathrm{P}<0,05)$ dos valores entre M4 e M36, assim como em M60, tendo os demais momentos se mantido próximos ao basal (Tab. 1).

Tabela 1. Médias \pm erro-padrão da média (EPM) da contagem de leucócitos totais $\left(\mathrm{x} 10^{3} / \mu \mathrm{L}\right)$, neutrófilos segmentados $\left(\times 10^{3} / \mu \mathrm{L}\right)$, neutrófilos bastonestes $\left(\times 10^{3} / \mu \mathrm{L}\right)$, linfócitos $\left(\times 10^{3} / \mu \mathrm{L}\right)$, eosinófilos $\left(\times 10^{3} / \mu \mathrm{L}\right)$, monócitos $\left(\times 10^{3} / \mu \mathrm{L}\right)$ e basófilos $\left(\times 10^{3} / \mu \mathrm{L}\right)$ no sangue venoso de equinos. Considera-se M0 antes do procedimento anestésico, M4 o momento de desobstrução intestinal e M12, M24, M36, M48, M60 e M72 como momentos pós-cirúrgicos

\begin{tabular}{|c|c|c|c|c|c|c|c|c|}
\hline & M0 & M4 & M12 & M24 & M36 & M48 & M60 & M72 \\
\hline \multirow{2}{*}{ Le } & $9,69 \pm$ & $9,79 \pm$ & $16,50 \pm$ & $13,70 \pm$ & $12,54 \pm$ & $12,31 \pm$ & $12,08 \pm$ & $10,79 \pm$ \\
\hline & $0,37^{\mathrm{c}}$ & $0,57^{\mathrm{c}}$ & $1,27^{\mathrm{a}}$ & $1,01^{\mathrm{ab}}$ & $1,15^{\mathrm{bc}}$ & $1,56^{\mathrm{bc}}$ & $1,49^{b c}$ & $1,10^{b c}$ \\
\hline \multirow[b]{2}{*}{ NS } & $5,36 \pm$ & $6,82 \pm$ & $13,00 \pm$ & $10,79 \pm$ & $9,58 \pm$ & $8,60 \pm$ & $8,71 \pm$ & $8,17 \pm$ \\
\hline & $0,49^{d}$ & $0,57^{\mathrm{cd}}$ & $1,12^{\mathrm{a}}$ & $1,17^{\mathrm{ab}}$ & $1,06^{b c}$ & $1,37^{\mathrm{bcd}}$ & $1,25^{\mathrm{bc}}$ & $1,07^{\mathrm{bcd}}$ \\
\hline \multirow{2}{*}{ NB } & $0,00 \pm$ & $0,03 \pm$ & $0,85 \pm$ & $0,23 \pm$ & $0,00 \pm$ & $0,00 \pm$ & $0,05 \pm$ & $0,09 \pm$ \\
\hline & $0,00^{\mathrm{c}}$ & $0,02^{b c}$ & $0,19^{\mathrm{a}}$ & $0,04^{b}$ & $0,00^{\mathrm{c}}$ & $0,00^{\mathrm{c}}$ & $0,03^{b c}$ & $0,04^{\mathrm{bc}}$ \\
\hline \multirow{2}{*}{$\mathrm{Ln}$} & $3,96 \pm$ & $2,67 \pm$ & $2,44 \pm$ & $2,47 \pm$ & $2,76 \pm$ & $3,22 \pm$ & $2,73 \pm$ & $2,93 \pm$ \\
\hline & $0,47^{\mathrm{a}}$ & $0,23^{b}$ & $0,40^{\mathrm{b}}$ & $0,38^{\mathrm{b}}$ & $0,29^{b}$ & $0,46^{\mathrm{ab}}$ & $0,35^{\mathrm{b}}$ & $0,43^{\mathrm{ab}}$ \\
\hline \multirow{2}{*}{ Eos } & $0,26 \pm$ & $0,15 \pm$ & $0,05 \pm$ & $0,02 \pm$ & $0,05 \pm$ & $0,28 \pm$ & $0,33 \pm$ & $0,30 \pm$ \\
\hline & $0,06^{\mathrm{a}}$ & $0,02^{\mathrm{ab}}$ & $0,05^{\mathrm{b}}$ & $0,02^{b}$ & $0,03^{b}$ & $0,08^{\mathrm{a}}$ & $0,11^{\mathrm{a}}$ & $0,10^{\mathrm{a}}$ \\
\hline \multirow{2}{*}{ Mo } & $0,14 \pm$ & $0,09 \pm$ & $0,15 \pm$ & $0,19 \pm$ & $0,14 \pm$ & $0,18 \pm$ & $0,21 \pm$ & $0,17 \pm$ \\
\hline & $0,03^{\mathrm{a}}$ & $0,03^{a}$ & $0,04^{\mathrm{a}}$ & $0,07^{\mathrm{a}}$ & $0,02^{\mathrm{a}}$ & $0,05^{\mathrm{a}}$ & $0,05^{\mathrm{a}}$ & $0,04^{\mathrm{a}}$ \\
\hline \multirow{2}{*}{ Bas } & $0,00 \pm$ & $0,02 \pm$ & $0,00 \pm$ & $0,00 \pm$ & $0,00 \pm$ & $0,03 \pm$ & $0,04 \pm$ & $0,04 \pm$ \\
\hline & $0,00^{\mathrm{a}}$ & $0,02^{\mathrm{a}}$ & $0,00^{\mathrm{a}}$ & $0,00^{\mathrm{a}}$ & $0,00^{\mathrm{a}}$ & $0,02^{\mathrm{a}}$ & $0,03^{\mathrm{a}}$ & $0,02^{\mathrm{a}}$ \\
\hline
\end{tabular}

Le (leucócitos totais), NS (neutrófilos segmentados), NB (neutrófilos bastonetes), Ln (linfócitos), Eos (eosinófilos), Mo (monócitos) e Bas (basófilos). Médias seguidas de mesmas letras na linha não diferem entre si (P>0,05). 
As MMP foram expressas em géis de zimograma (Fig. 1), por meio de bandas de diferentes pesos moleculares, expressas em quilodalton $(\mathrm{kDa})$, nos quais a MMP-2 na sua forma inativa (próMMP-2) foi detectada na maior parte das amostras de tecido laminar do casco, porém sem que houvesse diferença entre os momentos avaliados; já a sua forma ativa (MMP-2-ativa) foi detectada nos momentos M0 e M4 e em algumas amostras do momento M72, entretanto, assim como a pró-MMP-2, sem que houvesse diferença significativa entre os momentos.

A MMP-9 em sua forma inativa (pró-MMP-9) foi detectada em grande parte das amostras, porém sem que houvesse diferença significativa entre os momentos; já a sua forma ativa (MMP9-ativa) não foi identificada em nenhumas das amostras entre todos os momentos.

\section{DISCUSSÃO}

Levando-se em consideração que a pressão de $80 \mathrm{mmHg}$ exercida pela bola no lúmen intestinal ocluiu, ao menos parcialmente, os vasos sanguíneos responsáveis pela irrigação da parede intestinal, assim como comprometeu a drenagem venosa, acredita-se que houve significativa diminuição da perfusão tecidual no período em que a bola foi mantida inflada, o que concretiza a ocorrência de hipóxia local, assim como relatado por Gravena et al. (2016). Diante disso, pode-se dizer que a desobstrução intraluminal ocorrida em M4 promoveu a reperfusão do segmento acometido. A ocorrência desses fenômenos leva a crer que tenha, possivelmente, havido a lesão de reperfusão, mesmo que em baixa intensidade, no segmento experimentalmente obstruído (Rowe e White, 2002).

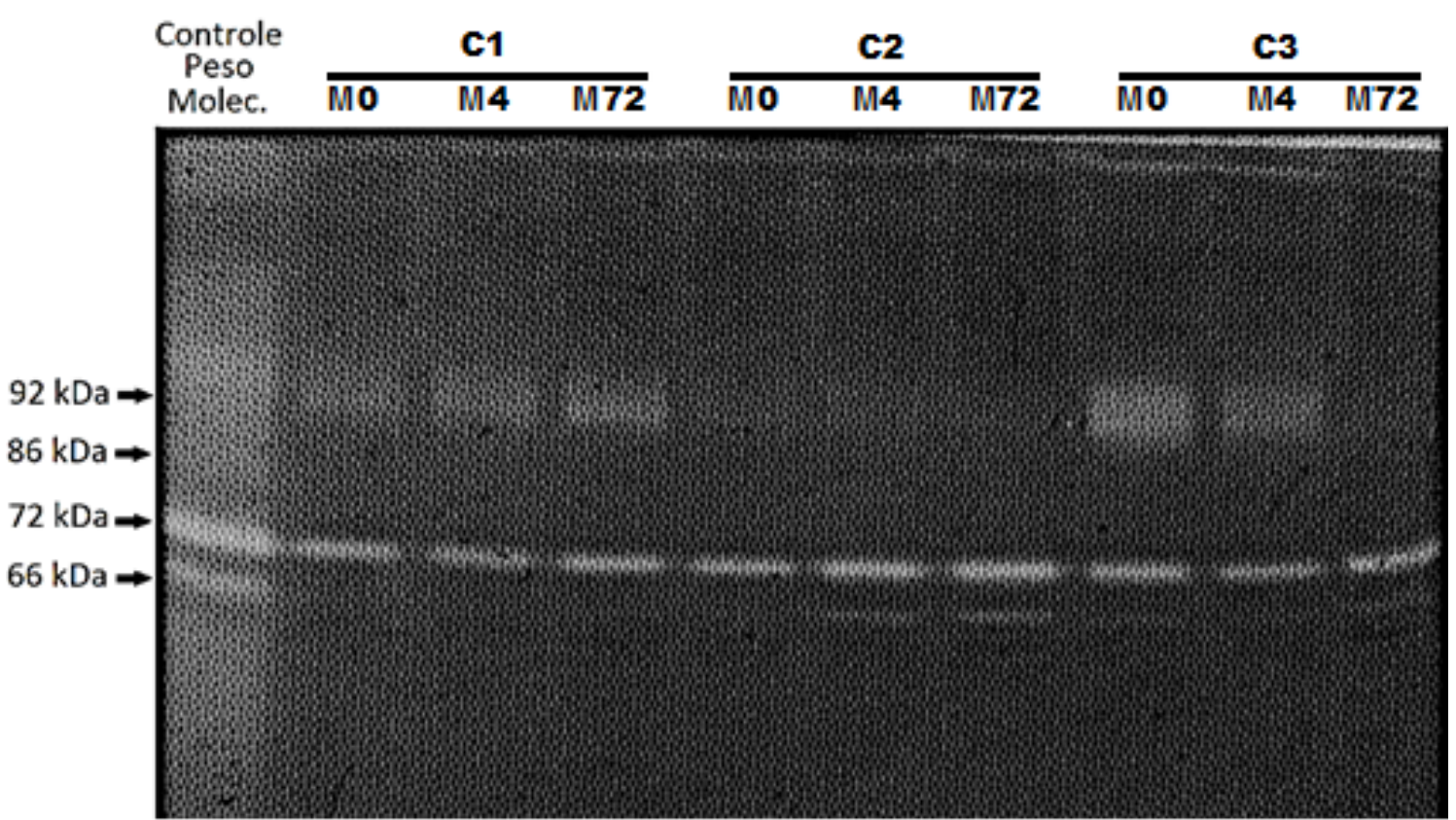

Figura 1. Zimograma representante das amostras de tecido laminar do casco, dos três momentos (M0, M4, M72) de três equinos submetidos à obstrução intraluminal do cólon menor (C1, C2, C3), no qual a primeira coluna é o controle de peso molecular (adenocarcinoma mamário canino). As bandas correspondem à pró-MMP-9 com peso molecular de $92 \mathrm{kDa}$, à MMP-9 ativa com $86 \mathrm{kDa}$, à pró-MMP-2 com $72 \mathrm{kda}$ e à MMP-2 ativa com 66kDa.

As lesões de reperfusão estão relacionadas ao restabelecimento da oferta de oxigênio aos tecidos antes em isquemia. Esse processo tem a participação de células endoteliais e receptores aferentes presentes no tecido lesionado, o que, por consequência, gera resposta inflamatória, inicialmente local, que pode evoluir para resposta sistêmica. Mudanças na membrana das células endoteliais causadas pelas lesões de isquemia e reperfusão levam à expressão de moléculas e receptores de adesão também relacionados à adesão e migração de neutrófilos 
através do endotélio de capilares e vênulas, consequentemente, provocando inflamação tecidual local mais pronunciada. Tendo em vista que a liberação sistêmica de citocinas e a ativação de neutrófilos circulantes podem levar a lesões em locais distantes à lesão inicial (White II, 2006), pode-se pressupor que a lesão causada pela obstrução intraluminal ocasionou, por consequência, a liberação sistêmica de mediadores inflamatórios, que, ao atingirem o tecido laminar do casco, poderiam acarretar o surgimento de lesões nesses tecidos, com possível aumento na expressão e ativação das MMP, que degradam a membrana basal presente na interface entre as lâminas epidérmicas secundárias e o tecido conectivo dérmico.

As análises leucocitárias revelaram aumento em M12 e M24, promovidas por polimorfonucleares (PMN) jovens e adultos, sendo a neutrofilia a responsável pela leucocitose de M12. Essas alterações são classificadas como leucocitose com desvio à esquerda regenerativo $\mathrm{e}$ provavelmente estão relacionadas à progressão da inflamação (Carrick e Begg, 2008).

A linfopenia e a eosinopenia observadas em M4 provavelmente estão relacionadas com o início da resposta inflamatória (Weiss e Wardrop, 2010) e também podem estar associadas à liberação de corticoides decorrentes do estresse cirúrgico (Carrick e Begg, 2008).

Autores como Faleiros (2003) observaram linfopenia durante obstrução intraluminal em equinos. Já Di Filippo (2009) observou eosinopenia em um único momento durante a fase inicial de reperfusão. Essas diferenças no leucograma em determinados momentos, quando comparadas ao presente trabalho, possivelmente estão associadas à diferença na intensidade da lesão ocasionada nos estudos.

Os casos em que a condição inflamatória do organismo se desenvolve por meio do sistema circulatório para os diferentes órgãos são conhecidos como síndrome da resposta inflamatória sistêmica (SIRS). Essa síndrome ocorre por estimulo excessivo de mediadores pró-inflamatórios, além de diversos outros estímulos infecciosos e não infecciosos, como, por exemplo, as lesões decorrentes de isquemia e reperfusão (Salles et al.,1999). A SIRS é caracterizada, entre vários fatores, por apresentar sinais como taquicardia, taquipneia, febre e leucocitose, até choque e falência múltipla dos órgãos nos casos mais graves. O diagnóstico da SIRS, em geral, baseia-se na presença de pelo menos duas dessas alterações citadas acima (Sherwood e Traber, 2012). Desse modo, no presente estudo, pode-se dizer que os animais estavam clinicamente em SIRS, pois apresentavam taquicardia, hipertermia e leucocitose em M12, condição mantida até M24. A partir de M36, os animais não apresentavam os sinais clínicos característicos da SIRS descritos por Sherwood e Traber (2012), condizente com a obstrução de grau leve.

Neste trabalho, as análises zimográficas não evidenciaram alterações nos valores da MMP-2 em sua forma ativa, ao contrário do encontrado por outros autores, como Loftus et al. (2009) e Johnson et al. (1998), que observaram aumento nos valores dessa enzima em equinos com laminite. Os valores da pró-MMP-2 também não se alteraram entre os diferentes momentos estudados. Possivelmente as alterações nos valores da MMP-2 em suas formas ativas e inativas não foram detectadas devido à baixa intensidade das lesões ocasionadas pelo método de obstrução.

Em relação à MMP-9, Johnson et al. (1998) e Loftus et al. (2009) observaram significativo aumento da forma inativa desta, ao contrário do relatado por Medina-Torres et al. (2011), que, em seu estudo, observaram diminuição nos valores dessa pró-enzima. Já no presente estudo, os valores da pró-MMP-9 não obtiveram alterações significativas. Tendo em vista que a expressão da pró-MMP-9 depende da presença de citocinas pró-inflamatórias, as quais atraem células inflamatórias para o tecido laminar, levando à expressão e ativação dessa MMP (Clutterbuck et al., 2010), pode-se também pressupor que o grau de lesão ocasionado pelo método de obstrução intestinal intraluminal utilizado neste estudo, apesar de ter causado isquemia parcial no segmento obstruído, foi de baixa intensidade, sendo insuficiente para ocasionar aumento na expressão da pró-MMP-9 nos tecidos laminares. 


\section{CONCLUSÃO}

A indução experimental de obstrução intraluminal do cólon menor não foi suficiente para levar a alterações nos valores de pró-MMP2, MMP-2-ativa, pró-MMP-9 e MMP-9-ativa, no tecido laminar do casco, possivelmente devido à baixa intensidade das lesões ocasionadas pelo método de obstrução utilizado. Entretanto, estudos complementares acerca da liberação de mediadores inflamatórios sistêmicos, que levam à ocorrência de lesões e à liberação e ativação das MMP no tecido laminar do casco equino, devem ser realizados para melhor compreensão do assunto.

\section{AGRADECIMENTOS}

À Fundação de Apoio à Pesquisa do Estado de São Paulo (Fapesp), pelo apoio financeiro e institucional.

\section{REFERÊNCIAS}

BELKNAP, J.K.; BLACK, S.J. Sepsis-related laminitis. Equine Vet. J., v.44, p.738-740, 2012.

CARRICK, A.P.; BEGG, A.P. Peripheral blood leukocytes. Vet. Clin. N. Am. Equine Pract., v.24, p.239-259, 2008.

CLUTTERBUCK, A.L.; HARRIS, P.; ALLAWAY, D. et al. Matrix metalloproteinases in inflammatory pathologies of the horse. Vet. J., v.183, p.27-38, 2010.

CORRÊA，R.R.; ZOPPA， A.L.V.; SILVA, L.C.L.C. et al. Estudo retrospectivo dos casos de enterolitíase e corpo estranho em intestino grosso de equinos, no período de janeiro de 1993 a janeiro de 2003. Braz. J. Vet. Res. Anim. Sci., v.43, p.242-249, 2006.

DI FILIPPO, P.A. Obstrução intestinal experimental em equinos: parâmetros clínicos $e$ laboratoriais. 2009. 112f. Tese (Doutorado em Cirurgia Veterinária) - Faculdade de Ciências Agrárias e Veterinárias, Universidade Estadual Paulista "Júlio de Mesquita Filho", Jaboticabal, SP.

FALEIROS, R.R. Obstrução experimental do cólon menor equino: aspectos clínicos, patológicos e terapêuticos. 2003. 168p. Tese (Doutorado em Cirurgia Veterinária) - Faculdade de Ciências Agrárias e Veterinárias, Universidade Estadual Paulista "Júlio de Mesquita Filho", Jaboticabal, SP.
FALEIROS, R.R.; MACORIS, D.G.; ALESSI, A.C. et al. Effect of intraluminal distention on microvascular perfusion in the equine small colon. Am. J. Vet. Res., v.63, p.1292-1297, 2002.

FALEIROS, R.R.; MACORIS, D.G.; ALVES, G.E.S. et al. Local and remote lesions in horses subjected to small colon distension and decompression. Can. J. Vet. Res., v.72, p.68-76, 2008.

GRAVENA, K.; HERNANDEZ-TOVAR, M.C.; CANELLO, V.A. et al. Clinical and laboratorial changes in horses subjected to a high-pressure modified model of small colon distention. $J$. Equine Vet. Sci., v.42, p.32-38, 2016.

GRAVENA, K.; SAMPAIO, R.C.L.; DIAS, D.P.M. et al. Evaluation of the integrity of horse hoof dermal and epidermal tissues collected by dorsal transmural access. J. Equine Vet. Sci., v.32, p.858-862, 2012.

JOHNSON, P.J.; TYAGI, S.C.; KATWA, L.C. et al. Activation of extracellular matrix metalloproteinases in equine laminitis. Vet. Rec., v.142, p.392-396, 1998.

LOFTUS, J.P.; JOHNSON, P.J.; BELKNAP, J.K. et al. Leukocyte-derived and endogenous matrix metalloproteinases in the lamellae of horses with naturally acquired and experimentally induced laminitis. Vet. Immunol. Immunopathol., v.129, p.221-230, 2009.

MACHADO, G.F.; MELO, G.D.; MORAES, O.C. et al. Differential alterations in the activity of matrix metalloproteinases within the nervous tissue of dogs in distinct manifestations of visceral leishmaniasis. Vet. Immunol. Immunopathol., v.136, p.340-345, 2010.

MAGDESIAN, K.G.; SMITH, B.P. Cólica. In: SMITH, B.P. Medicina interna de grandes animais. 3.ed. Barueri: Manole, 2006. p.108-111.

MEDINA-TORRES, C.E.; MASON, S.L.; FLOYD, R.V. et al. Hypoxia and a hypoxia mimetic up-regulate matrix metalloproteinase 2 and 9 in equine laminar keratinocytes. Vet. J., v.190, p.54-59, 2011.

MOORE, R.M.; MUIR, W.W.; GRANGER, D.N. Mechanisms of gastrointestinal ischemiareperfusion injury and therapeutic interventions: a review and its implications in the horses. J. Vet. Intern. Med., v.9, p.115-132, 1995. 
MUNGALL, B.A.; POLLITT C.C. Zymographic analysis of equine laminitis. Histochem. Cell Biol., v.112, p.467-472, 1999.

POLLITT, C.C. Anatomy and physiology of the inner hoof wall. Clin. Tech. Equine Pract., v.3, p.3-21, 2004.

POLLITT, C.C. Clinical anatomy and physiology of the normal equine foot. Vet. Cont. Educ., v.129, p.1-47, 1990.

POLLITT, C.C.; PASS, M.A.; POLLITT, S. Batimastat (BB-94) inhibits matrix metalloproteinases of equine laminitis. Equine Vet. J., v.26, p.119-124, 1998.

RAFFETTO, J.D.; KHALIL， R.A. Matrix metalloproteinases and their inhibitors in vascular remodeling and vascular disease. Biochem. Pharmacol., v.75, p.346-359, 2008.

ROWE, E.L.; WHITE, N.A. Repefusion injury in the equine intestine. Clin. Tech. Equine Pract., v.1, p.148-162, 2002.

SALLES, M.J.C.; SPROVIERI, S.R.S.; BEDRIKOW, R. et al. Síndrome da resposta inflamatória sistêmica/sepse-revisão e estudo da terminologia e fisiopatologia. Rev. Assoc. Med. Bras., v.45, p.86-92, 1999.
SHERWOOD, E.R.; TRABER, D.L. The systemic inflammatory response syndrome. In: HERNDON, D.N. Total burn care. 4.ed. Philadelphia: Saunders Elsevier, 2012. p.249263.

VISSE, R.; NAGASE, H. Matrix metalloproteinases and tissue inhibitors of metalloproteinases: structure, function, and biochemistry. Circ. Res., v.92, p.827-839, 2003.

VISSER, M.B.; POLLITT, C.C. The timeline of metalloprotease events during oligofructose induced equine laminitis development. Equine Vet. J., v.44, p.88-93, 2011.

VU, T.H.; WERB, Z. Matrix metalloproteinases: effectors of development and normal physiology. Genes Dev., v.14, p.2123-2133, 2000.

WEISS, D.J.; WARDROP, K.J. Interpretation of equine leukocyte responses. In: (Eds.). Schalm's veterinary hematology. 6.ed. Iowa: Wiley-Blackwell, 2010. p.314-320.

WHITE II, N.A. O intestine equino. In: SMITH, B.P. Medicina interna de grandes animais. 3.ed. Barueri: Manole, 2006. p.625-629.

WOESSNER Jr, J.F. Matrix metalloproteinases and their inhibitors in connective tissue remodeling. FASEB. J., v.5, p.2145-2154, 1991. 